\title{
Effects of Calcium and Vitamin D Supplementation on Crypt Morphology in Normal Colon Mucosa: A Randomized Clinical Trial
}

\author{
Huafeng Shen ${ }^{1}$, Thomas U. Ahearn ${ }^{1}$, and Roberd M. Bostick ${ }^{1,2,{ }^{*}}$ \\ ${ }^{1}$ Department of Epidemiology, Rollins School of Public Health, Emory University, Atlanta, Georgia \\ ${ }^{2}$ Winship Cancer Institute, Emory University, Atlanta, Georgia
}

\begin{abstract}
Calcium and vitamin D modify the molecular phenotypic profiles of colon crypts in the normal colorectal mucosa of colorectal adenoma patients, but their effects on crypt morphology (length, perimeter, and area) are unknown. We analyzed data from a previously conducted pilot, randomized, double-blind, placebo-controlled $2 \times 2$ factorial chemoprevention clinical trial of supplemental calcium $2000 \mathrm{mg} / \mathrm{d}$ and vitamin $\mathrm{D}_{3} 800 \mathrm{IU} / \mathrm{d}$, alone and in combination, versus placebo over 6 mo. Colorectal crypt length, perimeter, and area in the normal-appearing rectal mucosa were quantified by image analysis. The mean crypt length increased by $1 \%(P=0.92)$ in the calcium group, and decreased by $2 \%(P=0.69)$ and $4 \%(P=0.40)$ in the vitamin D and calcium plus vitamin $\mathrm{D}$ groups, respectively, relative to the placebo group. The mean crypt perimeter decreased by $2 \%(P=0.70)$ and $4 \%(P=0.40)$ in the vitamin $\mathrm{D}$ and calcium plus vitamin $\mathrm{D}$ groups, respectively, relative to the placebo group, but did not change appreciably in the calcium group. The mean crypt area decreased by $2 \%(P=0.74), 5 \%(P=0.41)$ and $7 \%(P=$ 0.30 ) in the calcium, vitamin $\mathrm{D}$ and calcium plus vitamin $\mathrm{D}$ groups, respectively, relative to the placebo group. Calcium and/or vitamin $\mathrm{D}_{3}$ supplementation do not appear to appreciably change crypt morphology in the normal colorectal mucosa of sporadic adenoma patients. These results, taken together with previous findings, support the use of molecular phenotypic over morphologic pre-neoplastic biomarkers of risk for colorectal neoplasms.
\end{abstract}

\section{Keywords}

crypt morphology; crypt length; randomized controlled trial; normal colorectal mucosa

\section{INTRODUCTION}

There is strong biological plausibility and animal experimental evidence for protection against colorectal cancer (CRC), the second leading cause of cancer deaths in the United States [1,2], by calcium and vitamin D. Proposed mechanisms of calcium against CRC include protection of colonocytes against bile acids and fatty acids [3], direct effects on cell

\footnotetext{
*Correspondence to: Roberd M. Bostick, Department of Epidemiology, 1518 Clifton Road NE, Atlanta, GA 30322. 
cycle regulation, modulation of the adenomatous polyposis coli (APC) colon carcinogenesis pathway, and modulation of E-cadherin and $\beta$-catenin expression via the calcium-sensing receptor [4-6]. Proposed mechanisms for vitamin D involve bile acid catabolism, direct effects on the cell cycle, growth factor signaling, cell adhesion, DNA repair and modulation of $>200$ genes $[4,7]$.

From the same preliminary trial reported herein, we previously reported changes in the expression of biomarkers of proliferation, apoptosis, differentiation, DNA mismatch repair, the APC/ $\beta$-catenin pathway, oxidative DNA damage, and calcium and vitamin D metabolism in the normal colorectal mucosa in response to calcium or vitamin $\mathrm{D}_{3}$ supplementation [8-13]. Crypt length, perimeter, and area are related to colonic cell proliferation, apoptosis, and differentiation, and, therefore, may serve as more simply measured, modifiable biomarkers of risk for colorectal neoplasms. Crypt length in the normal colon mucosa was inhibited by calcium in an animal study, but not in a small, uncontrolled trial $(n=17)$ or a randomized controlled trial $(n=111)$ [14-16]. There have been no previous trials of vitamin $\mathrm{D}$ alone or co-used with calcium on colon crypt morphology in humans. To clarify whether calcium and/or vitamin $\mathrm{D}_{3}$ affect crypt morphologic characteristics (length, perimeter, and area) in the normal human colorectal mucosa, we analyzed data from a previously conducted pilot, randomized, double-blind, placebo-controlled $2 \times 2$ factorial chemoprevention clinical trial [8-13].

\section{PATIENTS AND METHODS}

\section{Participant Population}

The detailed protocol for recruitment procedures and specific exclusions was published previously [8]. Eligibility included age 30-75 yr, in general good health, with a history of at least one pathology-confirmed sporadic colon or rectal adenoma within the past $36 \mathrm{mo}$, and no contraindications to calcium or vitamin D supplementation or rectal biopsy procedures and no medical conditions, habits, or medication usage that would otherwise interfere with the study. Participants were recruited from the patient population attending the Digestive Diseases Clinic of Emory University. The Emory University Institutional Review Board approved this study.

\section{Clinical Trial Protocol}

Between April 2005 and January 2006, 522 eligible patients were identified after initial chart screening, and $224(43 \%)$ potentially eligible patients were sent an introductory letter followed by a telephone interview. A total of $105(47 \%)$ potential participants attended an eligibility visit during which they were interviewed, signed a consent form, completed questionnaires, and provided a blood sample. Diet was assessed with a semi-quantitative food frequency questionnaire [17]. Medical and pathology records were reviewed. After a 30-d placebo run-in trial, 92 (88\%) eligible participants with no significant perceived side effects and who took at least $80 \%$ of their assigned tablets underwent a baseline rectal biopsy and were randomly assigned to the following four treatment groups ( $n=23 /$ treatment group): placebo, $2.0 \mathrm{~g}$ elemental calcium supplementation (as calcium carbonate in equal doses twice daily), $800 \mathrm{IU}$ vitamin $\mathrm{D}_{3}$ supplementation (400 IU twice daily), and $2.0 \mathrm{~g}$ 
elemental calcium plus $800 \mathrm{IU}$ vitamin $\mathrm{D}_{3}$ supplementation. Additional details on the rationale for the doses and forms of calcium and vitamin $\mathrm{D}$ supplementation forms were described previously [8]. Over the 6-mo treatment period, participants attended follow-up visits 2 and 6 mo after randomization. At each follow-up visit, participants were interviewed and completed questionnaires about adherence and adverse events, and at the final follow-up visit, underwent venipuncture and a rectal biopsy procedure. Plasma 25-OH-vitamin D and 1,25- $(\mathrm{OH})_{2}$-vitamin D were measured at baseline and 6-mo follow up using a radioimmunoassay method [8].

\section{Tissue Collection and Processing}

Six 1-mm-thick biopsy specimens were taken from the rectal mucosa $10 \mathrm{~cm}$ proximal to the external anal aperture through a rigid sigmoidoscope with a jumbo cup flexible endoscopic forceps mounted on a semi-flexible rod. The biopsies were placed onto a strip of bibulous paper and immediately placed in PBS, oriented under a dissecting microscope, placed in $10 \%$ normal buffered formalin, and then transferred to $70 \%$ ethanol $24 \mathrm{~h}$ after initial placement in formalin. Then, within a week, the biopsies were processed and embedded in paraffin blocks with three biopsies per block. The paraffin blocks were cut into 3.0- $\mu \mathrm{m}$-thick sections. Five slides with four section levels each taken $40 \mu \mathrm{m}$ apart were prepared, yielding a total of 20 levels per patient per visit.

\section{Image Analysis of the Crypt Morphology (Length, Perimeter, and Area of Crypts) in Normal Colon Crypts}

A quantitative image analysis method ("scoring") was used to evaluate the colonic crypt morphology (length, perimeter, and area of crypts), as shown in Figure 1. A "scorable" hemicrypt was defined as an intact hemicrypt that extended from the muscularis mucosa to the colon lumen. The major equipment and software for the image analysis procedures were a Scanscope CS digital scanner (Aperio Technologies, Inc., CA), computer, digital drawing board, Matlab software (Math-Works, Inc., MA), CellularEyes Image Analysis Suite (DivEyes, LLC, GA), and MySQL (Sun Microsystems, Inc., CA). The detailed protocol was previously described [8-13]. The goal was to analyze a minimum of 16 hemicrypts per each biopsy visit. One slide reader analyzed all slides throughout the study. Blinded subsets of previously analyzed slides were resubmitted to the reader during the study to assess intrareader reliability, which was found to be 0.98 for crypt length, 0.98 for crypt perimeter, 0.95 for crypt area, and 0.94 for the number of crypts scored.

\section{Statistical Analysis}

Primary analyses were based on assigned treatment at the time of randomization, regardless of adherence status (intent-to-treat analysis). Treatment effects were evaluated by assessing the differences in length, perimeter, and area of crypts from baseline to the 6-mo follow-up between participants in each active treatment group and those in the placebo group by a repeated-measures linear MIXED effects model. The model included the intercept, followup visit effects (baseline and follow-up), and interactions between treatment groups and the follow-up visit effect (the absolute treatment effect). To provide perspective on the magnitude of the treatment effects, we also calculated relative effects, defined as [(treatment 
group follow-up mean)/(treatment group baseline mean)]/[(placebo follow-up mean)/

(placebo baseline mean)].

Statistical analyses were performed using SAS 9.2 statistical software (SAS Institute, Inc., NC). A cutoff $P$ value $₫ 0.05$ (two-sided) was considered statistically significant.

\section{RESULTS}

\section{Characteristics of Study Participants}

Treatment groups did not differ significantly on characteristics measured at baseline or follow up, as published previously [8]. The mean age of participants was $61 \mathrm{yr}, 70 \%$ were men, $70 \%$ were white, $15 \%$ reported taking nonsteroidal anti-inflammatory drugs (NSAIDs) at least once a week, and $21 \%$ had a family history of CRC in a first-degree relative. Most participants were nonsmokers and college graduates, and, on average, tended to be overweight. Baseline serum 25-OH-vitamin D and 1,25-(OH $)_{2}$-vitamin D levels did not differ among the four treatment groups. On average, at least $80 \%$ of pills were taken by $93 \%$ of participants at the first follow-up visit and by $84 \%$ of participants at the final follow-up visit, and did not significantly differ among the four treatment groups [8]. By the conclusion of the study, serum 25-OH-vitamin D levels increased 60\% $(P<0.0001)$ and 56\% ( $P$ $<0.0001)$ in the vitamin $\mathrm{D}_{3}$ and calcium/ vitamin $\mathrm{D}_{3}$ groups, respectively, relative to placebo, whereas, as expected, there were no changes in 1,25-(OH $)_{2}$-vitamin D levels or in the calcium group [8].

\section{Effects of Calcium and/or Vitamin $\mathrm{D}_{3}$ Supplementation on Crypt Morphology (Length, Perimeter, and Area of Crypts) in Normal Colorectal Crypts}

Crypt length, perimeter, and area were strongly correlated at baseline, with $r=1.00(P$ $<0.0001)$ between crypt length and perimeter, $r=0.86(P<0.0001)$ between crypt length and area, and $r=0.88(P<0.0001)$ between crypt perimeter and area. The clinical trial crypt morphology endpoint results are shown in Table 1. At baseline, the four treatment groups did not differ significantly in crypt length, perimeter, or area. After 6 mo of treatment, the mean crypt length, perimeter, and area did not change significantly in any of the three active treatment groups, relative to the placebo group. In our preliminary findings, the effect of treatment on crypt length did not vary by sex, age, or NSAID use, as summarized in Table 2.

\section{DISCUSSION}

The results of this pilot randomized, controlled clinical trial provide the first evidence on the effects of supplemental calcium and vitamin $\mathrm{D}_{3}$, alone or jointly, on crypt morphology (length, perimeter, and area) in the normal colorectal mucosa of sporadic adenoma patients. Although in the same preliminary trial changes in the expression of biomarkers of proliferation, apoptosis, differentiation, DNA mismatch repair, the APC/ $\beta$-catenin pathway, oxidative DNA damage, and calcium and vitamin D metabolism were found in the normal colorectal mucosa [8-13], in the current analysis we found no evidence to suggest that calcium and/or vitamin D supplementation substantially changes crypt morphology in the normal colorectal mucosa. It also appeared that the effect of treatment on crypt length did not vary by sex, age, or NSAID use. The findings from this study are consistent with the 
results of two previous, randomized, controlled trials, which indicated that calcium supplementation did not affect the overall proliferation rate in the colorectal mucosa of sporadic adenoma patients $[9,18,19]$.

There are several possible explanations for the null findings. The first is that calcium and/or vitamin D simply do not meaningfully affect crypt morphology. Second, is chance, especially considering the small sample size. A third possibility is that our vitamin D dose may have been too low. The optimal levels of serum 25-(OH)-vitamin D are suggested to be $33-100 \mathrm{ng} / \mathrm{mL}$. To achieve these serum vitamin D levels in industrialized countries with increasing indoor lifestyles, total vitamin D exposures requires 1000-4000 IU/d [20]. Evidence from a large clinical trial of colorectal adenoma recurrence suggested that calcium supplementation was primarily effective among those with $25-(\mathrm{OH})$-vitamin D levels greater than the cohort median $(29.1 \mathrm{ng} / \mathrm{mL})$ [20]. In our trial, the vitamin $\mathrm{D}_{3}$ supplementation groups reached mean $25-(\mathrm{OH})$-vitamin D levels of only approximately $29 \mathrm{ng} / \mathrm{mL}$ at 6-mo follow-up [8]. This suggests that vitamin D supplementation at $800 \mathrm{IU} / \mathrm{d}$ may not yield a sufficient serum vitamin D level to affect crypt morphology in the normal colorectal mucosa.

There are only a few animal and human studies on the effects of calcium on crypt length in the normal colon mucosa. In a transmissible murine colonic hyperplasia (TMCH) model, diet with $1.0 \%$ calcium and $6 \%$ pectin inhibited increases in crypt length compared with the standard diet with $0.5 \%$ calcium and $5 \%$ cellulose [14]. In a small uncontrolled intervention study $(n=17)$, no change in crypt length was observed after $12 \mathrm{wk}$ and $1 \mathrm{yr}$ of $1500 \mathrm{mg}$ calcium supplementation [15]. In a randomized controlled trial $(n=111)$, no differences in crypt length were observed in four colorectal sites (cecum, transverse colon, sigmoid colon, and rectum) after $2 \mathrm{mo}$ of calcium $1000 \mathrm{mg}$ supplementation [16]. There have been no previous trials of vitamin $\mathrm{D}$ alone or combined with calcium on colon crypt morphology in humans.

This study had several strengths and limitations. The most obvious limitation was the small sample size, which may have increased the probability of chance findings in detecting or not detecting a treatment effect. Also, crypt morphometrics are not proven biomarkers of risk for colon cancer. Another limitation was that treatment effects could not be examined in parts of the colon other than the rectum. The effects of vitamin D alone or in combination with calcium on crypt morphology in different parts of the colon (other than the rectum) are not clear, as there have been no such studies in humans. On the other hand, this study is the first randomized, double-blind, placebo-controlled trial to have assessed the independent and combined effects of supplemental calcium and vitamin D on crypt morphology in the normal colorectal epithelium, protocol adherence by study participants was high, novel quantitative image analysis procedures were used, and biopsy analysis reliability was high.

Overall, although in the same preliminary trial changes in the expression of biomarkers of proliferation, apoptosis, differentiation, DNA mismatch repair, oxidative DNA damage, the APC/ $\beta$-catenin pathway, and calcium and vitamin D metabolism were found in the normal colorectal mucosa [8-13], the current analysis suggests that supplemental calcium and vitamin $\mathrm{D}_{3}$, individually or together, may not change the crypt morphology (length, 
perimeter, and area) in the normal human colorectal epithelium of sporadic adenoma patients.

\section{Acknowledgments}

Grant sponsor: National Cancer Institute; Grant sponsor: National Institutes of Health; Grant number: R01 CA104637; Grant sponsor: The Franklin Foundation; Grant sponsor: The Georgia Cancer Coalition

This work was supported by National Cancer Institute, National Institutes of Health (R01 CA104637 to R.M.B.); Georgia Cancer Coalition Distinguished Scholar award (to R.M.B.); the Franklin Foundation. The National Cancer Institute, the Georgia Cancer Coalition, and the Franklin Foundation had no influence on the design of the study; the collection, analysis, and interpretation of the data; the decision to submit the manuscript for publication; or the writing of the manuscript.

\section{Abbreviations}
CRC
colorectal cancer
NSAIDs
nonsteroidal anti-inflammatory drugs

\section{References}

1. Siegel R, Naishadham D, Jemal A. Cancer statistics, 2013. CA Cancer J Clin. 2013; 63:11-30. [PubMed: 23335087]

2. Potter JD, Slattery ML, Bostick RM, Gapstur SM. Colon cancer: A review of the epidemiology. Epidemiol Rev. 1993; 15:499-545. [PubMed: 8174669]

3. Newmark HL, Lipkin M. Calcium, vitamin D, and colon cancer. Cancer Res. 1992; 52:2067s2070s. [PubMed: 1544142]

4. Lamprecht SA, Lipkin M. Chemoprevention of colon cancer by calcium, vitamin D and folate: Molecular mechanisms. Nat Rev Cancer. 2003; 3:601-614. [PubMed: 12894248]

5. Chakrabarty S, Wang H, Canaff L, Hendy GN, Appelman H, Varani J. Calcium sensing receptor in human colon carcinoma: Interaction with $\mathrm{Ca}(2+)$ and 1,25-dihydroxyvitamin $\mathrm{D}(3)$. Cancer Res. 2005; 65:493-498. [PubMed: 15695391]

6. Rodland KD. The role of the calcium-sensing receptor in cancer. Cell Calcium. 2004; 35:291-295. [PubMed: 15200153]

7. Ebert R, Schutze N, Adamski J, Jakob F. Vitamin D signaling is modulated on multiple levels in health and disease. Mol Cell Endocrinol. 2006; 248:149-159. [PubMed: 16406653]

8. Fedirko V, Bostick RM, Flanders WD, et al. Effects of vitamin D and calcium supplementation on markers of apoptosis in normal colon mucosa: A randomized, double-blind, placebo-controlled clinical trial. Cancer Prev Res (Phila). 2009; 2:213-223. [PubMed: 19258546]

9. Fedirko V, Bostick RM, Flanders WD, et al. Effects of vitamin D and calcium on proliferation and differentiation in normal colon mucosa: A randomized clinical trial. Cancer Epidemiol Biomarkers Prev. 2009; 18:2933-2941. [PubMed: 19861511]

10. Sidelnikov E, Bostick RM, Flanders WD, et al. Effects of calcium and vitamin D on MLH1 and MSH2 expression in rectal mucosa of sporadic colorectal adenoma patients. Cancer Epidemiol Biomarkers Prev. 2010; 19:1022-1032. [PubMed: 20332274]

11. Fedirko V, Bostick RM, Long Q, et al. Effects of supplemental vitamin D and calcium on oxidative DNA damage marker in normal colorectal mucosa: A randomized clinical trial. Cancer Epidemiol Biomarkers Prev. 2010; 19:280-291. [PubMed: 20056649]

12. Ahearn TU, McCullough ML, Flanders WD, et al. A randomized clinical trial of the effects of supplemental calcium and vitamin D3 on markers of their metabolism in normal mucosa of colorectal adenoma patients. Cancer Res. 2011; 71:413-423. [PubMed: 21084270] 
13. Umar S, Morris AP, Kourouma F, Sellin JH. Dietary pectin and calcium inhibit colonic proliferation in vivo by differing mechanisms. Cell Prolif. 2003; 36:361-375. [PubMed: 14710853]

14. Kleibeuker JH, Welberg JW, Mulder NH, et al. Epithelial cell proliferation in the sigmoid colon of patients with adenomatous polyps increases during oral calcium supplementation. Br J Cancer. 1993; 67:500-503. [PubMed: 8439500]

15. van Gorkom BA, Karrenbeld A, van der Sluis T, et al. Calcium or resistant starch does not affect colonic epithelial cell proliferation throughout the colon in adenoma patients: A randomized controlled trial. Nutr Cancer. 2002; 43:31-38. [PubMed: 12467132]

16. Willett WC, Sampson L, Browne ML, et al. The use of a self-administered questionnaire to assess diet four years in the past. Am J Epidemiol. 1988; 127:188-199. [PubMed: 3337073]

17. Bostick RM, Fosdick L, Wood JR, et al. Calcium and colorectal epithelial cell proliferation in sporadic adenoma patients: A randomized, double-blinded, placebo-controlled clinical trial. J Natl Cancer Inst. 1995; 87:1307-1315. [PubMed: 7658483]

18. Baron JA, Tosteson TD, Wargovich MJ, et al. Calcium supplementation and rectal mucosal proliferation: A randomized controlled trial. J Natl Cancer Inst. 1995; 87:1303-1307. [PubMed: 7658482]

19. Bostick, RM.; Goodman, M.; Sidelnikov, E. Calcium and vitamin D. In: Potter, JD.; Lindor, NM., editors. Genetics of colorectal cancer. New York, NY: Springer; 2009. p. 277-298.

20. Grau MV, Baron JA, Sandler RS, et al. Vitamin D, calcium supplementation, and colorectal adenomas: Results of a randomized trial. J Natl Cancer Inst. 2003; 95:1765-1771. [PubMed: 14652238] 
A

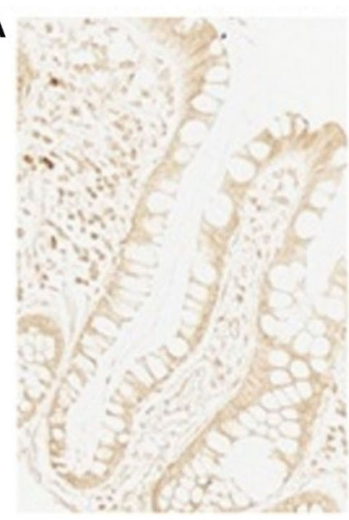

B

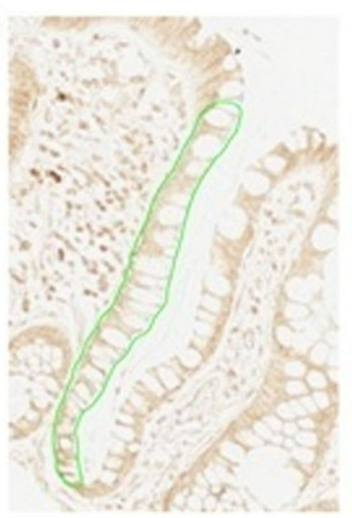

C

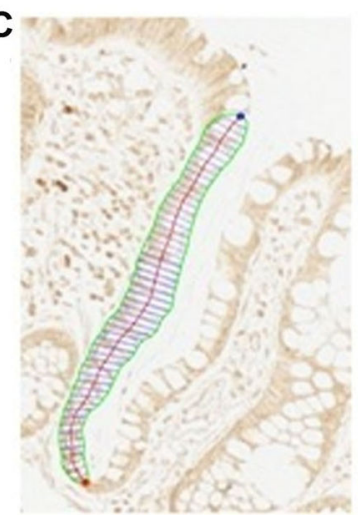

Figure 1.

(modified from Ref. [12]). Quantitative image analysis using Aperio Scanscope and CellularEyes software to measure crypt length, perimeter, and area in normal-appearing colorectal mucosa. (A) Choosing scorable crypts; (B) tracing borders of hemicrypt; (C) automated quantification of crypt length, perimeter, and area. 


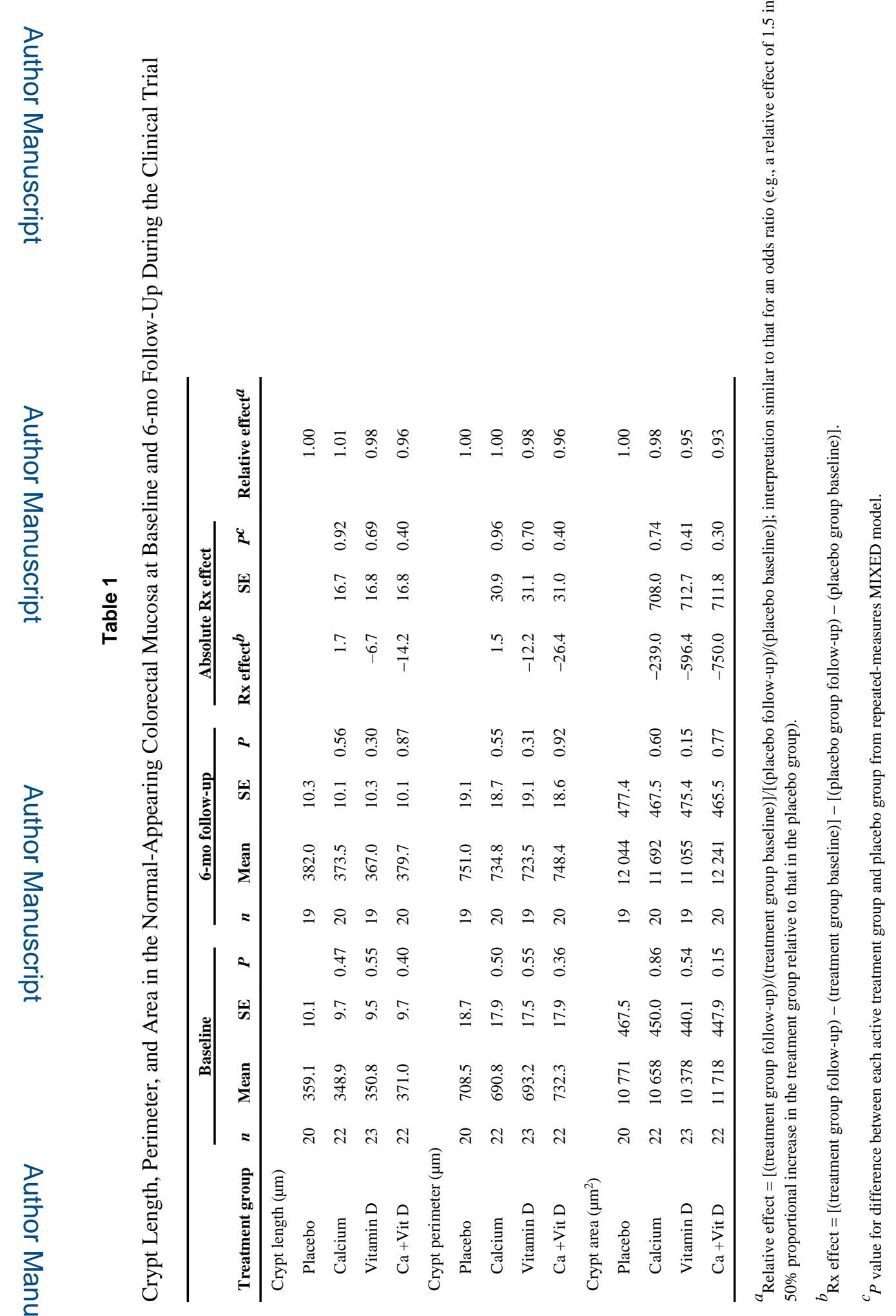

Mol Carcinog. Author manuscript; available in PMC 2016 March 01. 


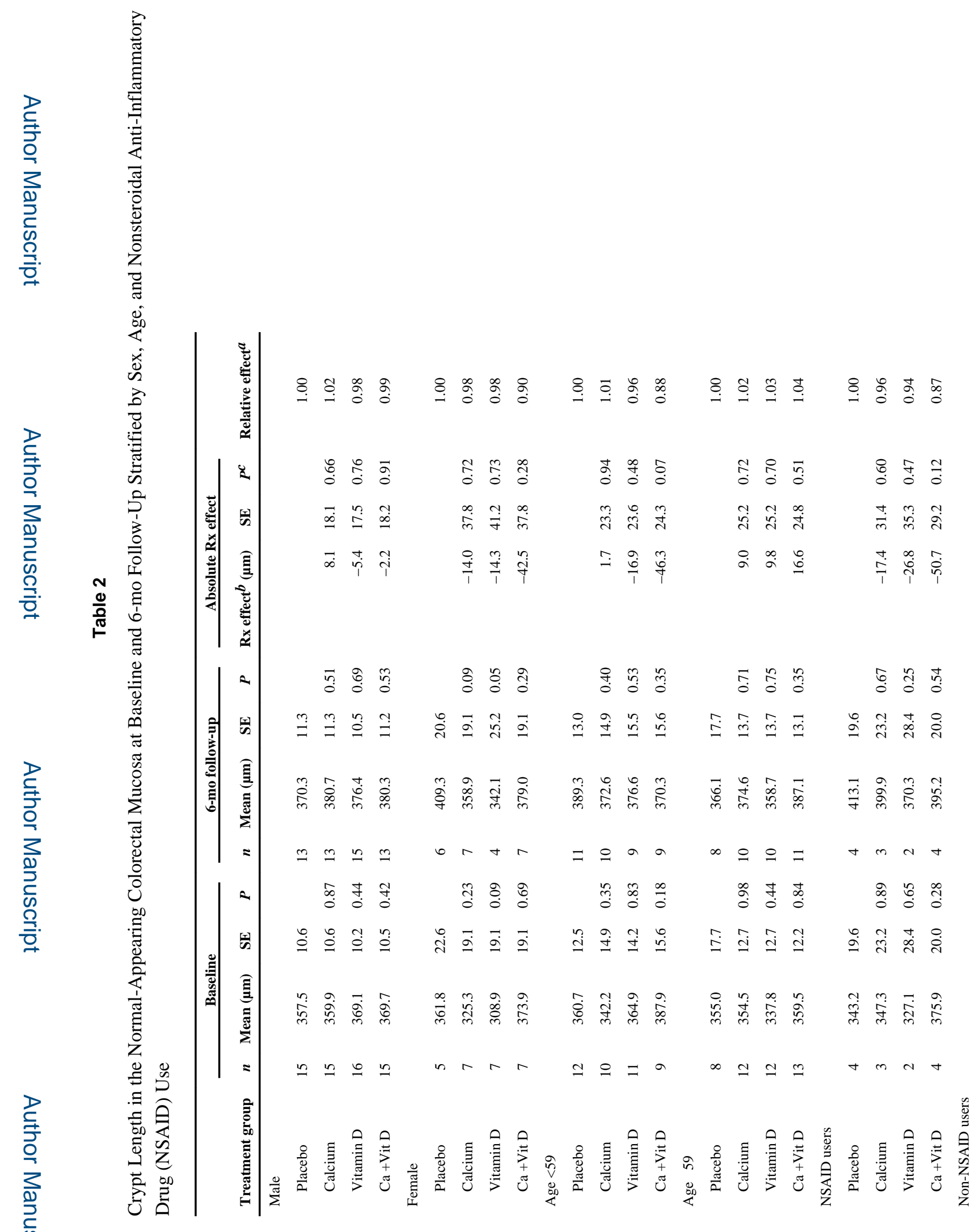

Mol Carcinog. Author manuscript; available in PMC 2016 March 01. 


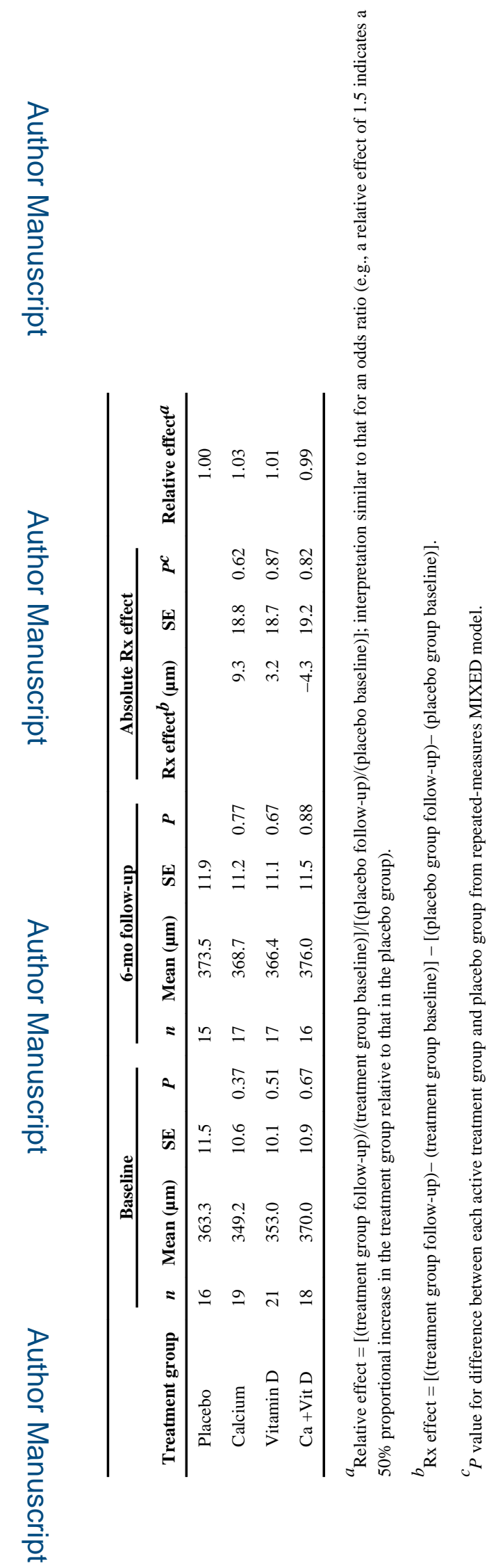

Mol Carcinog. Author manuscript; available in PMC 2016 March 01. 